\title{
Lactate rewires synovial T cells in RA
}

The inflamed synovium in rheumatoid arthritis (RA) contains a high concentration of lactate that has traditionally been thought to be a by-product of synovial fibroblast proliferation. In a new study, researchers describe how lactate can function as an effector molecule by reprogramming $\mathrm{CD} 4^{+}$ $\mathrm{T}$ cells to a pro-inflammatory phenotype, thereby exacerbating disease in patients with RA who predominantly have lymphoid cell infiltrates in their synovium (the so-called lymphoid pathotype).

"We identified the pathway initiated by lactate build-up in inflamed tissue that exacerbates the tissue inflammatory response in diseases with an important $\mathrm{CD}^{+} \mathrm{T}$ cell component, such as the lymphoid pathotype of RA," states corresponding author Claudio Mauro. Specifically, the researchers investigated SLC5A12, a sodium-dependent lactate transporter protein that they have previously shown to be expressed on $\mathrm{CD}^{+}{ }^{+} \mathrm{T}$ cells, but not $\mathrm{CD} 8^{+}$

$\mathrm{T}$ cells, in mice. In their new study, the researchers showed that, upon activation with either anti-CD3 antibodies or RA synovial fluid, SLC5A12 was upregulated in peripheral blood $\mathrm{CD} 4^{+} \mathrm{T}$ cells from patients with RA compared with those from healthy individuals.

Further in vitro and in vivo experiments revealed that lactate was taken up by SLC5A12 in CD4 $4^{+}$ $\mathrm{T}$ cells, where it induced a switch in metabolism away from glycolysis and towards fatty acid synthesis and PMK2 nuclear activity. These changes in metabolism led to the increased expression of IL-17 by these cells in the presence of lactate. Experiments with synovial tissue explants also revealed a role for SLC5A12 in controlling lactate-induced tissue retention of T cells; blocking SLC5A12 with an antibody enabled the egress of $\mathrm{CD}^{+} \mathrm{T}$ cells from the tissue explants.

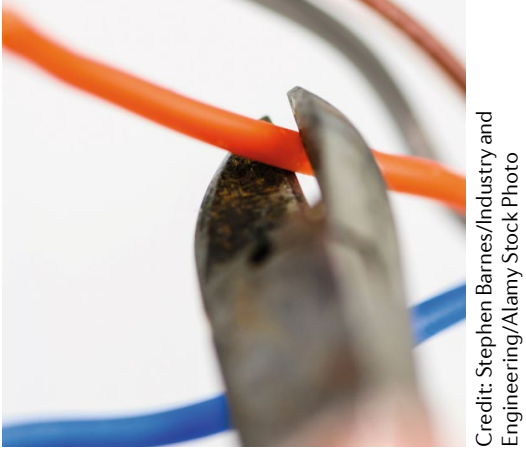

RNA sequencing of synovial tissue samples from DMARD-naive patients with RA showed a distinct positive correlation between SLC5A12 expression and disease activity. "We also used a murine model of RA that is characterized by $\mathrm{CD} 4^{+} \mathrm{T}$ cell infiltration in the arthritic joints to mimic the lymphoid pathotype and showed that blocking SLC5A12 improves the clinical signs of disease," says Mauro. The researchers suggest that SLC5A12 could be a future therapeutic target for the lymphoid pathotype of RA.

Joanna Clarke induced a switch in metabolism
ORIGINAL ARTICLE Pucino, V. et al. Lactate buildup at the site of chronic inflammation promotes disease by inducing $\mathrm{CD} 4^{+} \mathrm{T}$ cell metabolic rewiring. Cell Metabolism https:// doi.org/10.1016/j.cmet.2019.10.004 (2019)

\section{New genetic risk loci found for SSc}

The results of the largest genomewide association study (GWAS) to date in patients with systemic sclerosis (SSc) have been published in Nature Communications. The study combined data from 14 independent European cohorts and revealed several new risk loci related to fibrosis and vasculopathy.

"Thanks to a large international collaborative effort, we gathered $\sim 10,000$ patients to perform a powerful large-scale genomic analysis of this rare condition," states co-corresponding author Elena López-Isac. "We have identified 27 independent genomewide associated signals, including 13 new risk loci, almost doubling the number of genome-wide hits reported for SSc thus far."

"The study also provides robust evidence to nominate the target genes behind the genetic associations," adds co-corresponding author Javier Martin. "Some of them point towards molecular pathways linked to vasculopathy (like DDX6, the ability of which to

seven of the identified genes encoded pharmacological targets

כ)
been previously demonstrated), which is involved in transforming regulate vascular endothelial growth factor under hypoxic conditions has or to fibrosis (like the role of TWSG1, growth factor- $\beta$ signalling in T cells)."

After identifying candidate risk loci in the GWAS screen, the authors used a combination of functional analysis and epigenomic techniques across a variety of cell types to investigate the most relevant genetic associations for the disease. In particular, transcription regulatory functions were uncovered for many genetic variants, and a strong signal for many associations was seen in NK cells, which have been previously linked to SSc pathogenesis.

Importantly, a subtype analysis revealed two unique associations, one for limited cutaneous SSc (MERTK, encoding a tyrosine kinase involved in liver fibrosis) and one for diffuse cutaneous SSc (ANKRD12, the associated variant of which is linked to TWSG1). "For the first time, we identified subtype-specific associated loci outside of the MHC region," says López-Isac. The authors suggest that the association of fibrotic pathways with diffuse cutaneous SSc is particularly interesting owing to the aggressive nature of this disease subtype.

The authors also performed a drug target enrichment analysis and found that seven of the identified genes encoded pharmacological targets. Therapeutics are already in clinical trials for SSc for two of these targets (CD80 and BLK), and the authors suggest that the presence of IL12A in the list of genes suggests that ustekinumab might be a candidate to be repurposed for SSc.

Joanna Clarke

ORIGINAL ARTICLE López-Isac, E. et al. GWAS for systemic sclerosis identifies multiple risk loci and highlights fibrotic and vasculopathy pathways. Nat. Commun. 10, 4955 (2019)

RELATED ARTICLE Angiolilli, C. et al. New insights into the genetics and epigenetics of systemic sclerosis. Nat. Rev. Rheumatol. 14, 657-673 (2018) 